\title{
Effect of local cold-pack application on systemic anabolic and inflammatory response to sprint-interval training: a prospective comparative trial
}

\author{
Dan Nemet $\cdot$ Yoav Meckel $\cdot$ Sheli Bar-Sela • \\ Frank Zaldivar · Dan M. Cooper · Alon Eliakim
}

Accepted: 16 July 2009 / Published online: 4 August 2009

(C) The Author(s) 2009. This article is published with open access at Springerlink.com

\begin{abstract}
We evaluated the effect of cold ice-pack application following a brief sprint-interval training on the balance between anabolic mediators [growth hormone $(\mathrm{GH})$, insulin-like growth factor-I (IGF-I), testosterone], catabolic markers (cortisol, IGFBP-1), and circulating pro [Interlukin-6 (IL-6) and IL-1 $\beta$ ]- and anti-inflammatory cytokines [IL-1 receptor antagonist (IL-1ra)]. Twelve males, elite junior handball players performed $4 \times 250 \mathrm{~m}$ treadmill run, at $80 \%$ of each individual's maximal speed, followed by a rest period with and without local cold-pack application. Pre, immediately post, and 60-min post-exercise blood samples were drawn. Exercise was associated with a significant increase in IL-6, GH, IGFBP-3, and testosterone levels. Local cold-pack application was associated with significant decreases in IL-1 $\beta$, IL-1ra, IGF-I, and IGFBP-3 and a greater increase of IGFBP-1 during recovery. Local ice therapy immediately following sprint-interval training was associated with greater decreases in both pro- and antiinflammatory cytokines and anabolic hormones supporting some clinical evidence for possible negative effects on athletic performance.
\end{abstract}

D. Nemet $(\bowtie) \cdot$ A. Eliakim

Pediatric Department,

Meir Medical Center, Child Health \& Sport Center,

Sackler School of Medicine, Tel-Aviv University,

Kfar-Saba 44821, Israel

e-mail: dnemet@gmail.com

D. Nemet · F. Zaldivar · D. M. Cooper - A. Eliakim Department of Pediatrics, Pediatric Exercise Research Center, University Children's Hospital, University California, Irvine, CA, USA

Y. Meckel · S. Bar-Sela · A. Eliakim

Zinman College of Physical Education,

Wingate Institute, Netanya, Israel
Keywords Cryotherapy $\cdot$ Anabolic $\cdot$ Inflammation · Recovery $\cdot$ Exercise $\cdot$ Cytokines

\section{Introduction}

Athletic success depends primarily on the combination of genetic endowment, training, technique, equipment, and proper nutrition (Rogol 2003). Thus, the use of additional aids in the training process is thought to play a relatively minor role in athletic performance. However, in competitive sports, where one hundredth of a second, or a few millimeters could make a difference between fame and shame in the life of an athlete, the search for legal methods to improve training ability and performance becomes critical.

The development of methods to enhance the recovery of elite athletes from intense training and/or competition has been a major target of athletes and their accompanying staff for many years. Cryotherapy is used widely to treat sportsassociated traumatic injuries and as a recovery modality following training and competition that may cause some level of traumatic muscle injury (Barnett 2006; Wilcock et al. 2006). However, evidence regarding the effectiveness and appropriate guidelines for the use of cryotherapy are limited. Several studies have shown that the use of cryotherapy in the recovery between training sessions reduced exercise-associated delayed onset muscle soreness (DOMS) (Lane and Wenger 2004; Yanagisawa et al. 2003), attenuated elevation in serum myoglobin concentrations, and reduced the post-exercise decrements in muscle strength (Bailey et al. 2007). In contrast, other studies indicated that post-exercise cryotherapy decreased trainingassociated adaptive processes and performance, and was not associated with decreased muscle damage or soreness 
(Howatson et al. 2005; Richendollar et al. 2006; Wassinger et al. 2007; Yamane et al. 2006).

Recent reports suggest that acute exercise may lead to significant changes in anabolic hormones and circulating inflammatory mediators. Surprisingly, exercise leads to a simultaneous increase in antagonistic mediators. On one hand, exercise stimulates anabolic components of the growth hormone $(\mathrm{GH}) \rightarrow$ insulin-like growth factor-I (IGF-I) axis (Schwarz et al. 1996; Stokes et al. 2005), while on the other hand, exercise increases catabolic pro-inflammatory cytokines such as Interlukin-6 (IL-6), IL-1, and tumor necrosis factor- $\alpha$ (TNF- $\alpha$ ) (Meckel et al. 2009; Nemet et al. 2002b). The assessment of changes in these antagonistic circulating mediators may assist in quantifying the effects of different types of exercise training and recovery modalities.

Therefore, the aim of this study is to assess the effect of local cold-pack application on the systemic response of anabolic hormones and inflammatory mediators to sprintinterval training in young elite handball players. Hormonal measurements included circulating levels of the anabolic hormones GH, IGF-I, IGF-binding protein-3 (IGFBP-3), and testosterone and the catabolic markers cortisol and IGFBP-1. Measurements of inflammatory mediators included the proinflammatory markers IL- 6 and IL- $1 \beta$, and the anti-inflammatory markers IL-1 receptor antagonist (IL-1ra) and IL-10. We hypothesized that local cold-pack application immediately post-exercise will reduce the training-associated systemic inflammatory process and, therefore, will lead to a decrease in pro-inflammatory cytokines and to a concomitant increase of the anabolic hormones. To the best of our knowledge, the effect of post-exercise cryotherapy on anabolic/ catabolic hormones and inflammatory mediators' response to exercise has not been previously studied.

\section{Subjects and methods}

Twelve healthy elite Israeli junior handball players (age range 17-20 years) participated in the study. All the participants played in the Israeli premier handball league, and some of the players belonged to the Israeli national junior handball team. The study was performed during the final stages of the regular handball season when the players are at their best physical shape. Training in this part of the season involved mainly tactic and technical drills emphasizing handball skills and team strategies, speed drills with and without the ball, and longer interval sessions (e.g., several repetitions of 20 - to 40 -s run at $\sim 80 \%$ of the maximal speed). No resistance training was done at the time period of the study. The study was approved by the Institutional Review Board of the Meir General Hospital, and informed consent was obtained from all the participants.
Table 1 Anthropometric characteristics of the study participants

\begin{tabular}{lc}
\hline Age (years) & $20.3 \pm 1.0$ \\
Body weight $(\mathrm{kg})$ & $74.5 \pm 2.3$ \\
Body height $(\mathrm{cm})$ & $179.7 \pm 2.1$ \\
BMI $\left(\mathrm{kg} / \mathrm{m}^{2}\right)$ & $23.1 \pm 0.8$ \\
Body fat $(\%)$ & $13.7 \pm 0.6$ \\
\hline
\end{tabular}

Anthropometric characteristics of the participants are summarized in Table 1. Standard, calibrated scales, and stadiometer were used to determine height and body mass, and BMI was calculated from these data. Skinfold measurement at four sites (triceps, biceps, sub-scapular, and supra-iliac) was used to estimate percent body fat using standard equations (Slaughter et al. 1988).

\section{Exercise and cryotherapy protocol}

Participants were instructed to avoid any physical activity, caffeine, and/or alcohol use at the day before each study. None of the participants used medications and/or dietary supplements at the time of the study.

Each participant performed twice an outdoors maximal 100-m run. The best result was used to calculate the speed of the interval exercise session. Exercise consisted of four 250-m runs on a treadmill (motor-driven treadmill; Woodway, PPS 55med, Weil am Rhein, Germany), at a constant intensity of $80 \%$ of the maximal speed (calculated from the speed of 100-m run), with 3-min rest between each of the $250-\mathrm{m}$ runs. This protocol was performed twice at random order, once without and once with local cold-pack application following the interval practice. The cold packs (half size 5 inch $\times 12$ inch Whitehall Glacier-Packs ${ }^{\mathrm{TM}}$ Reusable Cold Packs) were applied immediately following the final 250-m run using a compression wrap. The cold packs were applied to the hamstring muscles for $15 \mathrm{~min}$, followed by 15-min interval, and then again for additional $15 \mathrm{~min}$ while the athletes were resting supine in a quiet room. Room temperature was $21^{\circ} \mathrm{C}$. Following the session without coldpack application, the athletes rested in the same conditions for $1 \mathrm{~h}$.

Blood sampling and analysis

Tests were performed in the morning, following an overnight fast. An indwelling venous catheter was inserted 30 min prior to the first blood draw. Pre, immediately after the last $250-\mathrm{m}$ run, and $60-\mathrm{min}$ post the last $250-\mathrm{m}$ run (recovery) blood samples were drawn from the catheter (Fig. 1). Blood samples were immediately spun at $3,000 \mathrm{rpm}$, at $4^{\circ} \mathrm{C}$ for $20 \mathrm{~min}$. The serum was separated and stored at $-80^{\circ} \mathrm{C}$. All pre- and post-exercise specimens from 


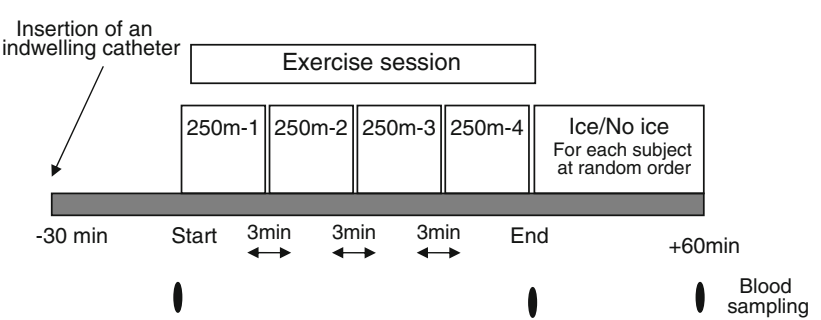

Fig. 1 Exercise protocol and blood sampling procedure. Each subject performed the protocol twice, once with and once without ice at the recovery period, at a random order

each individual were analyzed in the same batch by an experienced technician who was blinded to the order of samples and to the type of interval training.

\section{Growth hormone}

Growth hormone serum concentrations were determined by ELISA with the use of the DSL-10-1900 Active kit (Diagnostic System Laboratories, Webster, Texas). Intra-assay $\mathrm{CV}$ was 3.3-4.5\%, inter-assay CV was 5.5-12.9\%, and the sensitivity was $0.03 \mathrm{ng} / \mathrm{ml}$.

\section{Insulin-like growth factor-I}

Insulin-like growth factor-I was extracted from IGF-binding proteins (IGFBPs) by using the acid-ethanol extraction method. Serum IGF-I concentrations were determined by a two-site immunoradiometric assay by using the DSL-5600 Active kit (Diagnostic System Laboratories, Webster, TX). IGF-I intra-assay CV was $1.5-3.4 \%$ and the inter-assay CV was $3.7-8.2 \%$. Assay sensitivity was $0.8 \mathrm{ng} / \mathrm{ml}$.

\section{IGF-binding proteins}

IGF-binding proteins-1 was measured by a coated-tube immunoradiometric assay with the use of the DSL-10-7800 Active kit (Diagnostic System Laboratories). Intra-assay $\mathrm{CV}$ was $2-4 \%$, and inter-assay $\mathrm{CV}$ was $1.7-6.7 \%$. Assay sensitivity is $0.33 \mathrm{ng} / \mathrm{ml}$. IGFBP-3 serum concentrations were determined by ELISA with the use of the DSL 10-6600 Active kit (Diagnostic System Laboratories). Intra-assay $\mathrm{CV}$ was 7.3-9.6\%, inter-assay CV was $8.2-11.4 \%$, and the sensitivity was $0.04 \mathrm{ng} / \mathrm{ml}$.

\section{Cortisol}

Serum cortisol levels were determined by a commercial RIA (Diagnostic Products Corporation, Los Angeles, CA). The intra- and inter-assay $\mathrm{CV}$ were 3.2 and $6.8 \%$, respectively.

\section{Testosterone}

Testosterone serum concentrations were determined by ELISA with the use of the DSL commercial kit (Diagnostic System Laboratories, Webster, Texas). Intra-assay CV was $4.8-5.3 \%$, inter-assay CV was $2.8-4.9 \%$, and the sensitivity was $0.04 \mathrm{ng} / \mathrm{ml}$.

\section{Inflammatory mediators}

Inflammatory mediators were analyzed by ELISA, using the R\&D system Quantikine High Sensitivity commercial kits (R\&D system; Minneapolis, MN).

Interleukin-6 (IL-6) Intra-assay CV was 3.8-11.1\%, inter-assay $\mathrm{CV}$ was $7.1-29.5 \%$, and the sensitivity was $0.0094 \mathrm{pg} / \mathrm{ml}$.

Interleukin-1 beta $(I L-1 \beta) \quad$ Intra-assay CV was 1.6-4.0\%, inter-assay $\mathrm{CV}$ was $5.3-9.0 \%$, and the sensitivity was $0.059 \mathrm{pg} / \mathrm{ml}$.

Interleukin-1 receptor antagonist (IL-1ra) Intra-assay $\mathrm{CV}$ was 3.1-6.2\%, inter-assay CV was $4.4-6.7 \%$, and the sensitivity was $22 \mathrm{pg} / \mathrm{ml}$.

Interleukin-10 (IL-10) Intra-assay CV was 8.1-15.6\%, inter-assay $\mathrm{CV}$ was $6.6-8.2 \%$, and the sensitivity was $0.5 \mathrm{pg} / \mathrm{ml}$.

\section{Statistical analyses}

A two-way repeated measure ANOVA (with Bonferroni post hoc test) was used to assess the effect of exercise on circulating components of the GH-IGF-I axis and inflammatory mediators with time serving as the within group factor and post-exercise cold-pack application as the between group factor. Data are presented as mean \pm SEM. Significance was taken at $P \leq 0.05$.

\section{Results}

Characteristics of the study participants are summarized in Table 1. The effect of local cold-pack application on the inflammatory response to the sprint-interval training in young elite handball players is summarized in Table 2 and Fig. 2. There was no baseline between group differences in any of the measured variables (with and without cold-pack application). Exercise, in both conditions, was associated with a significant increase in IL-6 levels $(P=0.004)$. Level of IL-6 remained elevated $1 \mathrm{~h}$ after the end of exercise. Local cold-pack application had no effect on IL-6 
Table 2 The effect of local cold-pack application on the systemic anabolic/catabolic hormonal and inflammatory response to sprint-interval training in young elite handball players

\begin{tabular}{|c|c|c|c|c|c|c|}
\hline & \multicolumn{2}{|l|}{ Pre-exercise } & \multicolumn{2}{|l|}{ Post-exercise } & \multicolumn{2}{|l|}{ Recovery } \\
\hline & No ice at recovery & Ice at recovery & No ice at recovery & Ice at recovery & No ice & Ice \\
\hline IL- $1 \beta$ & $0.6 \pm 0.1$ & $1.2 \pm 0.3$ & $0.8 \pm 0.1$ & $1.5 \pm 0.4$ & $0.5 \pm 0.1$ & $0.8 \pm 0.3^{\mathrm{c}}$ \\
\hline IL-6 & $1.4 \pm 0.3$ & $1.4 \pm 0.2$ & $1.7 \pm 0.2^{\mathrm{a}}$ & $2.1 \pm 0.3^{\mathrm{a}}$ & $2.1 \pm 0.3^{\mathrm{a}}$ & $1.9 \pm 0.3^{\mathrm{a}}$ \\
\hline IL1ra & $331.6 \pm 46.8$ & $399.4 \pm 60.4$ & $353.0 \pm 73.8$ & $424.0 \pm 57.7$ & $332.1 \pm 66.1$ & $284.0 \pm 40.5^{\mathrm{c}}$ \\
\hline IL-10 & $0.7 \pm 0.6$ & $1.1 \pm 0.6$ & $0.7 \pm 0.3$ & $0.6 \pm 0.5$ & $0.9 \pm 0.6$ & $0.5 \pm 0.5$ \\
\hline $\mathrm{GH}$ & $0.4 \pm 0.3$ & $0.3 \pm 0.1$ & $5.7 \pm 2.6^{\mathrm{a}}$ & $5.8 \pm 2.7^{\mathrm{a}}$ & $0.3 \pm 0.2^{b}$ & $0.2 \pm 0.1^{\mathrm{b}}$ \\
\hline IGF-I & $350.2 \pm 45.9$ & $396.4 \pm 59.4$ & $383.0 \pm 40.0$ & $428.0 \pm 50.0$ & $375.1 \pm 35.8$ & $299.7 \pm 50.0^{c}$ \\
\hline IGFBP-3 & $4382 \pm 252$ & $4579 \pm 224$ & $4864 \pm 307^{\mathrm{a}}$ & $4886 \pm 229^{\mathrm{a}}$ & $4532 \pm 230$ & $4099 \pm 226^{c}$ \\
\hline IGFBP-1 & $15.5 \pm 3.7$ & $12.3 \pm 2.2$ & $15.4 \pm 4.4$ & $15.5 \pm 3.6$ & $18.3 \pm 2.5$ & $25.5 \pm 4.7^{\mathrm{c}}$ \\
\hline Testosterone & $4.3 \pm 0.3$ & $4.2 \pm 0.6$ & $4.9 \pm 0.5^{\mathrm{a}}$ & $4.8 \pm 0.6^{\mathrm{a}}$ & $4.1 \pm 0.4^{b}$ & $4.0 \pm 0.6^{\mathrm{b}}$ \\
\hline Cortisol & $19.3 \pm 2.3$ & $20.5 \pm 2.1$ & $17.6 \pm 1.9$ & $18.1 \pm 2.3$ & $13.8 \pm 1.1^{\mathrm{b}}$ & $12.6 \pm 1.9^{\mathrm{b}}$ \\
\hline
\end{tabular}

Data presented as mean \pm SEM

a Significant within group changes from pre-exercise values

b Significant within group changes from post-exercise values

c Significant between group differences (ice vs. no ice)
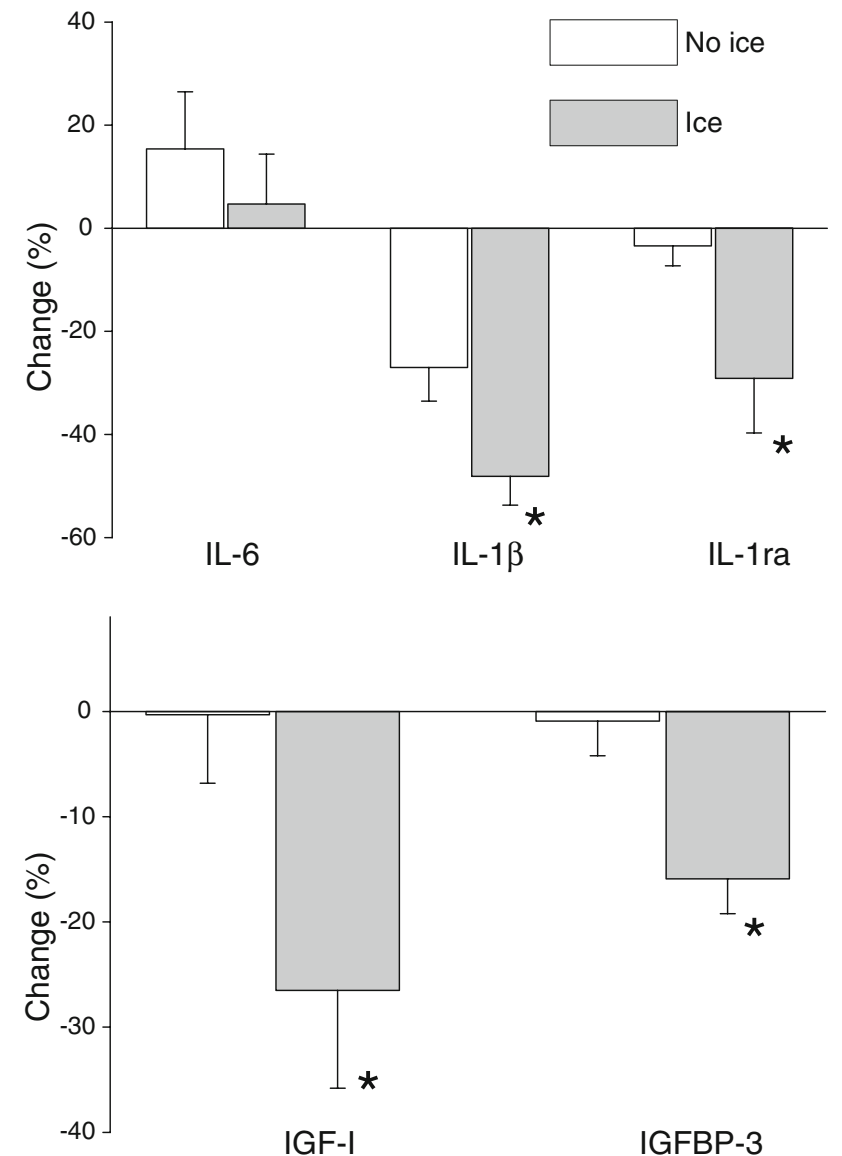

Fig. 2 The effect of local cold-pack application on recovery changes of the pro (IL-6, IL-1 $\beta$ )- and anti (IL-1ra)-inflammatory mediators (upper panel) and anabolic hormones (lower panel) from post-exercise levels. *Significant between group difference concentrations. Exercise was associated with a non-significant increase in IL-1 $\beta$. Local cold-pack application was associated with a significant greater decrease in IL- $1 \beta$ during the recovery from exercise $(P=0.04$; Table 2$)$. Exercise had no effect on IL-1ra levels. However, application of local cold packs resulted in a significant decrease of this anti-inflammatory mediator during the recovery from exercise $(P=0.03$; Table 2). Exercise and cold-pack application had no effect on IL-10 levels.

The effect of local cold-pack application on the systemic anabolic/catabolic response to the sprint-interval training in young elite handball players is summarized in Table 2 and Fig. 2. Exercise, in both conditions, was associated with a significant increase in GH $(P=0.048)$, IGFBP-3 $(P=0.003)$, and testosterone levels $(P=0.001)$. Local cold-pack application was associated with a significantly greater decrease of IGF-I $(P=0.043)$ and IGFBP-3 $(P=0.019)$ during the recovery from exercise. Local cold-pack application was also associated with a significantly greater increase in IGFBP-1 levels during the recovery from exercise $(P=0.01)$. There was no significant effect of exercise on cortisol levels. However, cortisol levels dropped significantly during the recovery from exercise $(P=0.005)$ with no effect of cold-pack application.

\section{Discussion}

Enhancing the recovery from intense training and/or competition has been a major goal of competitive athletes and their coaches. Cryotherapy has been widely used for many years as a recovery modality to reduce sports-associated 
major and minor traumatic injuries and training-induced muscular inflammation to improve recovery and to increase training capacity (Barnett 2006; Wilcock et al. 2006). The aim of this study is to assess the effect of local cold-pack application on systemic anabolic and catabolic hormonal adaptation and on circulating inflammatory cytokines response to a brief sprint-interval training in elite young handball players. The results of the study indicate that local cold-pack application was indeed associated with significant systemic hormonal and inflammatory effects. Local cold-pack application was associated with a significantly greater decrease of the pro-inflammatory mediator IL-1 $\beta$, but had no effect on circulating IL-6 levels. However, coldpack administration was also associated with even a greater decrease in the anti-inflammatory mediator IL-1ra during the recovery from exercise. In addition, and in contrast to our hypothesis, local cold-pack application immediately after the training session led to a significantly greater decrease in the circulating level of the anabolic hormones IGF-I and IGFBP-3, and to a significantly greater increase in the catabolic agent IGFBP-1.

The use of local cold ice is associated with a decrease in skin and tissue temperature. This leads to vasoconstriction of sympathetic fibers, reduces local blood flow and local metabolic rate and as a result, reduces tissue swelling, inflammation, pain sensation, and the degree of injury (Enwemeka et al. 2002). However, evidence on the effectiveness of ice-pack application to improve recovery following intense training is limited. There is no clear scientific evidence to support that apart from an analgesic effect, cryotherapy has a beneficial effect on the prevention or reduction of delayed onset muscle soreness (Cheung et al. 2003).

Cytokines play a key communicating role between immune functions and neuroendocrine adaptations, and therefore may influence immune homeostasis in cold environments (Castellani et al. 2002). IL-6, a cytokine believed to play an important mediatory role in the inflammatory response to exercise, usually increases following intense and prolonged exercise. However, previous reports indicated that IL-6 may also significantly increase (although to a lesser degree) following brief exercise, and even when only a small muscle group is involved (Nemet et al. 2002a). It was shown that prolonged cold-wet exposure following strenuous exercise differentially modulated cytokine production, up-regulating IL-6 and IL-1ra production and down-regulating IL- $1 \beta$ and TNF $\alpha$ secretion (Rhind et al. 2001). Moreover, cold exposure during exercise attenuates monocytes intracellular synthesis of proinflammatory cytokines like IL- $1 \beta$ and TNF $\alpha$, has no effect on IL-6 elevation and stimulates the expression of the antiinflammatory mediator IL-1ra (Castellani et al. 2002). Secretion of adreno-sympathetic hormones was correlated with both cold-associated circulating and intra-cellular cytokine changes (Castellani et al. 2002; Rhind et al. 2001).
Consistent with these previous reports, local cold-pack application immediately after exercise was associated with a significantly greater decrease of IL- $1 \beta$, and had no effect on IL-6 levels. However, in contrast to previous reports, postexercise ice application was also associated with a significant decrease in the anti-inflammatory marker IL-1ra. It is possible that differences in the type of exercise, length of ice exposure (brief in the present study and long in the previous reports), and the method of cold exposure (local vs. systemic) were responsible for the differences of IL-1ra response.

As was previously reported for anaerobic and aerobic exercise (Schwarz et al. 1996; Stokes et al. 2005), exercise was associated with a significant increase of anabolic hormones. These hormones returned to the pre-exercise levels during the recovery ( 1 hour after the end of exercise). Interestingly, the application of local cold pack immediately after exercise led to a significantly greater decrease in the circulating level of the anabolic hormones IGF-I and IGFBP-3, and to a significantly greater increase in the catabolic agent IGFBP-1. These results suggest that local administration of cryotherapy may, therefore, attenuate the anabolic effects of the previous training.

Several studies have demonstrated conflicting effects of cryotherapy on athletic performance. Bailey et al. (2007) have shown that the use of cryotherapy attenuated post-exercise elevation in serum myoglobin concentrations, and reduced the post-exercise decrements in muscle strength. The combination of ice treatment with light recovery exercise enhanced shoulder strength recovery from baseball pitching (Yanagisawa et al. 2003). Lane and Wenger (2004) reported that the combination of cold-water immersion with active recovery or massage (but not with rest) maintained work capacity when high-intensity exercise was repeated after $24 \mathrm{~h}$. In contrast, other studies indicated that post-exercise cryotherapy was not associated with faster strength recovery from eccentric exercise in resistance trained males (Howatson and van Someren 2008), and untrained males and females (Eston and Peters 1999; Howatson et al. 2005). Moreover, post-exercise cryotherapy reduced training-associated adaptive processes and performance in untrained men (Yamane et al. 2006). Ice-bag application was noted to negatively affect performance in a single-leg vertical jump, shuttle run time and 40-yard sprint time in young active males (Richendollar et al. 2006). Shoulder icebag application resulted in reduced proprioception and throwing accuracy in healthy college males and females (Wassinger et al. 2007). In this study, post-training local ice therapy reduced the anabolic (IGF-I and IGFBP-3) and increased the catabolic (IGFBP-1) hormonal response. This suggests that the application of cold pack immediately after exercise may result not only in performance decrement but also in attenuation of the anabolic effects of the preceding training.

Testosterone and cortisol levels are frequently measured to determine the anabolic/catabolic effects of training and 
recovery. Exercise was associated with an increase in testosterone levels. Testosterone levels returned to baseline in the recovery with no effect of the cold-pack application. Exercise was not associated with a significant change in cortisol level. This is not surprising since a significant increase in cortisol levels requires exercise duration of at least $20 \mathrm{~min}$ in an intensity of at least $60 \%$ of the maximal oxygen consumption (Urhausen and Kindermann 2000). Cortisol levels decreased following recovery to a lower than baseline levels. A possible explanation for this decrease is the diurnal circadian rhythm of cortisol, which leads to a decrease in cortisol levels during the day, compared to early morning levels. Cold-pack application had no effect on cortisol levels as well.

Several important methodological issues should be pointed out. First, we determined the effects of cold-pack application only $60 \mathrm{~min}$ after the end of exercise. The significant effects on anabolic, catabolic, and pro- and anti-inflammatory mediators indicate that longer effects of cold-pack application should be studied as well. Second, we studied the effect of local cold-pack application during the recovery from a brief sprint-interval training on systemic anabolic/catabolic and inflammatory adaptation in elite handball players. We used the same exercise protocol without cold-pack application during the recovery as a control. The results of this study suggests that in future studies, a control group to determine the effects of local cold-pack application at resting condition (without exercise), should be included as well. Thus, further studies are needed before conclusive practical recommendations regarding the anabolic, catabolic, and inflammatory effect of the use of coldpack application could be made.

In summary, local ice therapy immediately following sprint-interval training was associated with a greater decrease in both pro- and anti-inflammatory cytokines and with a greater decrease in anabolic hormones. These results, along with the previously reported evidence for some negative effects of ice application on athletic performance, and no clear effect on muscle damage or DOMS may suggest that the use of cold packs should probably be reserved for traumatic injuries or used in combination with active recovery or massage and not with complete rest.

Open Access This article is distributed under the terms of the Creative Commons Attribution Noncommercial License which permits any noncommercial use, distribution, and reproduction in any medium, provided the original author(s) and source are credited.

\section{References}

Bailey DM, Erith SJ, Griffin PJ, Dowson A, Brewer DS, Gant N, Williams C (2007) Influence of cold-water immersion on indices of muscle damage following prolonged intermittent shuttle running. J Sports Sci 25:1163-1170

Barnett A (2006) Using recovery modalities between training sessions in elite athletes: does it help? Sports Med 36:781-796

Castellani JW, Brennes IKM, Rhind SG (2002) Cold exposure: human immune responses and intracellular cytokine expression. Med Sci Sports Exerc 34:2013-2020

Cheung K, Hume P, Maxwell L (2003) Delayed onset muscle soreness: treatment strategies and performance factors. Sports Med 33:145-164

Enwemeka CS, Allen C, Avila P, Bina J, Konrade J, Munns S (2002) Soft tissue thermodynamics before, during, and after cold pack therapy. Med Sci Sports Exerc 34:45-50

Eston R, Peters D (1999) Effects of cold water immersion on the symptoms of exercise-induced muscle damage. J Sports Sci 17:231-238

Howatson G, van Someren KA (2008) The prevention and treatment of exercise-induced muscle damage. Sports Med 38:483-503

Howatson G, Gaze D, van Someren KA (2005) The efficacy of ice massage in the treatment of exercise-induced muscle damage. Scand J Med Sci Sports 15:416-422

Lane KN, Wenger HA (2004) Effect of selected recovery conditions on performance of repeated bouts of intermittent cycling separated by 24 hours. J Strength Cond Res 18:855-860

Meckel Y, Eliakim A, Seraev M, Zaldivar F, Cooper DM, Sagiv M, Nemet D (2009) The effect of a brief sprint interval exercise on growth factors and inflammatory mediators. J Strength Cond Res 23:225-230

Nemet D, Hong S, Mills PJ, Ziegler MG, Hill M, Cooper DM (2002a) Systemic vs. local cytokine and leukocyte responses to unilateral wrist flexion exercise. J Appl Physiol 93:546-554

Nemet D, Oh Y, Kim HS, Hill M, Cooper DM (2002b) Effect of intense exercise on inflammatory cytokines and growth mediators in adolescent boys. Pediatrics 110:681-689

Rhind SG, Castellani JW, Brenner IK, Shephard RJ, Zamecnik J, Montain SJ, Young AJ, Shek PN (2001) Intracellular monocyte and serum cytokine expression is modulated by exhausting exercise and cold exposure. Am J Physiol Regul Integr Comp Physiol 281:R66-R75

Richendollar ML, Darby LA, Brown TM (2006) Ice bag application, active warm-up, and 3 measures of maximal functional performance. J Athl Train 41:364-370

Rogol AD (2003) Dietary supplements to enhance athletic performance. In: Lifshitz F (ed) Pediatric endocrinology, 4th edn. Marcl Dekker, Inc., New York, pp 917-922

Schwarz AJ, Brasel JA, Hintz RL, Mohan S, Cooper DM (1996) Acute effect of brief low- and high-intensity exercise on circulating insulin-like growth factor (IGF) I, II, and IGF-binding protein-3 and its proteolysis in young healthy men. J Clin Endocrinol Metab 81:3492-3497

Slaughter MH, Lohman TG, Boileau RA, Horswill CA, Stillman RJ, Van Loan MD et al (1988) Skinfold equations for estimation of body fatness in children and youth. Hum Biol 60(5):709-723

Stokes K, Nevill M, Frystyk J, Lakomy H, Hall G (2005) Human growth hormone responses to repeated bouts of sprint exercise with different recovery periods between bouts. J Appl Physiol 99:1254-1261

Urhausen A, Kindermann W (2000) The endocrine system in overtraining. In: Warren MP, Constantini NW (eds) Sports endocrinology. Humana Press, Totowa, pp 347-370

Wassinger CA, Myers JB, Gatti JM, Conley KM, Lephart SM (2007) Proprioception and throwing accuracy in the dominant shoulder after cryotherapy. J Athl Train 42:84-89

Wilcock IM, Cronin JB, Hing WA (2006) Physiological response to water immersion: a method for sport recovery? Sports Med 36:747-765 
Yamane M, Teruya H, Nakano M, Ogai R, Ohnishi N, Kosaka M (2006) Post-exercise leg and forearm flexor muscle cooling in humans attenuates endurance and resistance training effects on muscle performance and on circulatory adaptation. Eur J Appl Physiol 96:572-580
Yanagisawa O, Miyanaga Y, Shiraki H, Shimojo H, Mukai N, Niitsu M, Itai Y (2003) The effects of various therapeutic measures on shoulder strength and muscle soreness after baseball pitching. J Sports Med Phys Fitness 43:189-201 